\title{
TAIWAN OSCILLATION NETWORK
}

\author{
DEAN-YI CHOU, MING-TSUNG SUN* and TENG-YI HUANG
}

Physics Department, Tsing Hua University, Hsinchu, 30043, Taiwan, R.O. China

ANTONIO JIMENEZ

Instituto Astrofisica de Canarias, Observatorio del Teide, Tenerife, Spain

SHIH-PING LAI, PI-JEN CHI, KNIGHT-TIEN OU, CHANG-CHI WANG, JUI-YANG LU, AN-LI CHU, CHI-SENG NIU, TAO-MO MU,

KUAN-RONG CHEN and YUNG-PING CHOU

Physics Department, Tsing Hua University, Hsinchu, 30043, Taiwan, R.O. China

HORANCE CHAO

Department of Electro-Optics Engineering, Lien-Ho Junior College of Technology, Miaoli, 36012, Taiwan, R.O. China

MARIA CRISTINA RABELLO-SOARES

Instituto Astrofisica de Canarias, Observatorio del Teide, Tenerife, Spain

GUOXIANG AI and GWO-PING WANG

Huairou Solar Observing Station, Beijing Observatory, Beijing, P.R. China

and

HAROLD ZIRIN, WILLIAM MARQUETTE and JEFF NENOW

Big Bear Solar Observatory, California Institute of Technology, Pasadena, CA 91125, U.S.A.

(Received 27 February, 1995; in revised form 18 April, 1995)

\begin{abstract}
The Taiwan Oscillation Network (TON) is a ground-based network to measure solar intensity oscillations to study the internal structure of the Sun. K-line full-disk images of 1000 pixels diameter are taken at a rate of one image per minute. Such data would provide information on $p$-modes with $l$ as high as 1000 . The TON will consist of six identical telescope systems at proper longitudes around the world. Three telescope systems have been installed at Teide Observatory (Tenerife), Huairou Solar Observing Station (near Beijing), and Big Bear Solar Observatory (California). The telescopes at these three sites have been taking data simultaneously since October of 1994. An $l-\nu$ diagram derived from 512 images is included to show the quality of the data.
\end{abstract}

\section{Introduction}

The Taiwan Oscillation Network (TON) is a ground-based network to measure solar K-line intensity oscillations to study the internal structure of the Sun. The TON project has been funded by the National Research Council of ROC since the summer of 1991. The TON will consist of six identical telescope systems deployed at proper longitudes around the world. The headquarters of the TON is located at the Physics Department of Tsing Hua University, Hsinchu, Taiwan, where the telescope systems are designed, built, and tested. The first telescope was installed at

* Department of Mechanical Engineering, Chang-Gung College of Medical and Technology, Kwei-San, Taiwan, R.O. China.

Solar Physics 160: 237-243, 1995.

(C) 1995 Kluwer Academic Publishers. Printed in Belgium. 
the Teide Observatory, Tenerife, Canary Islands, Spain in August 1993. The second one was installed at the Huairou Solar Observing Station near Beijing in January 1994. The third telescope system was installed at the Big Bear Solar Observatory, California in June 1994. The telescopes at these three sites have been taking data simultaneously since October 1994 . The last three telescope systems are expected to be installed in 1995 and 1996. The site selection and arrangement of the last three telescope systems are underway.

The TON is designed to obtain information on high-degree solar $p$-mode oscillations, along with intermediate-degree modes. The TON telescope system uses a 3.5-inch Maksutov-type telescope to observe K-line full-disk solar images with a 16-bit $1080 \times 1080$ water-cooled CCD. The solar images are taken and saved on magnetic tapes at a rate of one image per minute. The diameter of the Sun is set to 1000 pixels. The measured amplitude of intensity oscillations is about $2.5 \%$. With the TON data one can study solar $p$-modes with spherical harmonic degree, $l$, as high as 1000 .

The TON images are similar to the K-line images obtained at the south pole by NSO (Duvall et al., 1993), and at Alaska and Hawaii by the University of Hawaii (Ronan and LaBonte, 1994). The TON is complementary to other existing and on-going ground-based network, such as BISON, IRIS, and GONG. The BISON (Elsworth et al., 1994) and the IRIS (Fossat, 1991) measure the integrated velocity. The GONG measures the imaging velocity and intensity with a solar diameter of about 220 pixels (Harvey and the GONG Instrument Development Team, 1988).

In Section 2 we will describe the hardware and controlling software of the TON. In Section 3, we briefly discuss the scientific goal of the TON project, In Section 4, we give the preliminary results from 512 images obtained with the TON telescope at Tenerife.

\section{Instrumentation}

The TON telescope system is semi-automated. In the morning, observers have to open the telescope cover, point the telescope to the Sun, and enter control commands on computers. Then the telescope will guide itself, and images will be taken and saved on magnetic tapes at a rate of one image per minute. The whole system is controlled by a PC-486, running UNIX. Another PC-486, running DOS, is used to monitor and display the brightness of the Sun and the temperatures of circulating water, $\mathrm{CCD}$ head, and the $\mathrm{CCD}$ control box. In the following, we will briefly describe the hardware and controlling software of a TON telescope system. The overall structure of a TON telescope system is best described by a block diagram shown in Figure 1. 


\section{TON System Block Diagram}

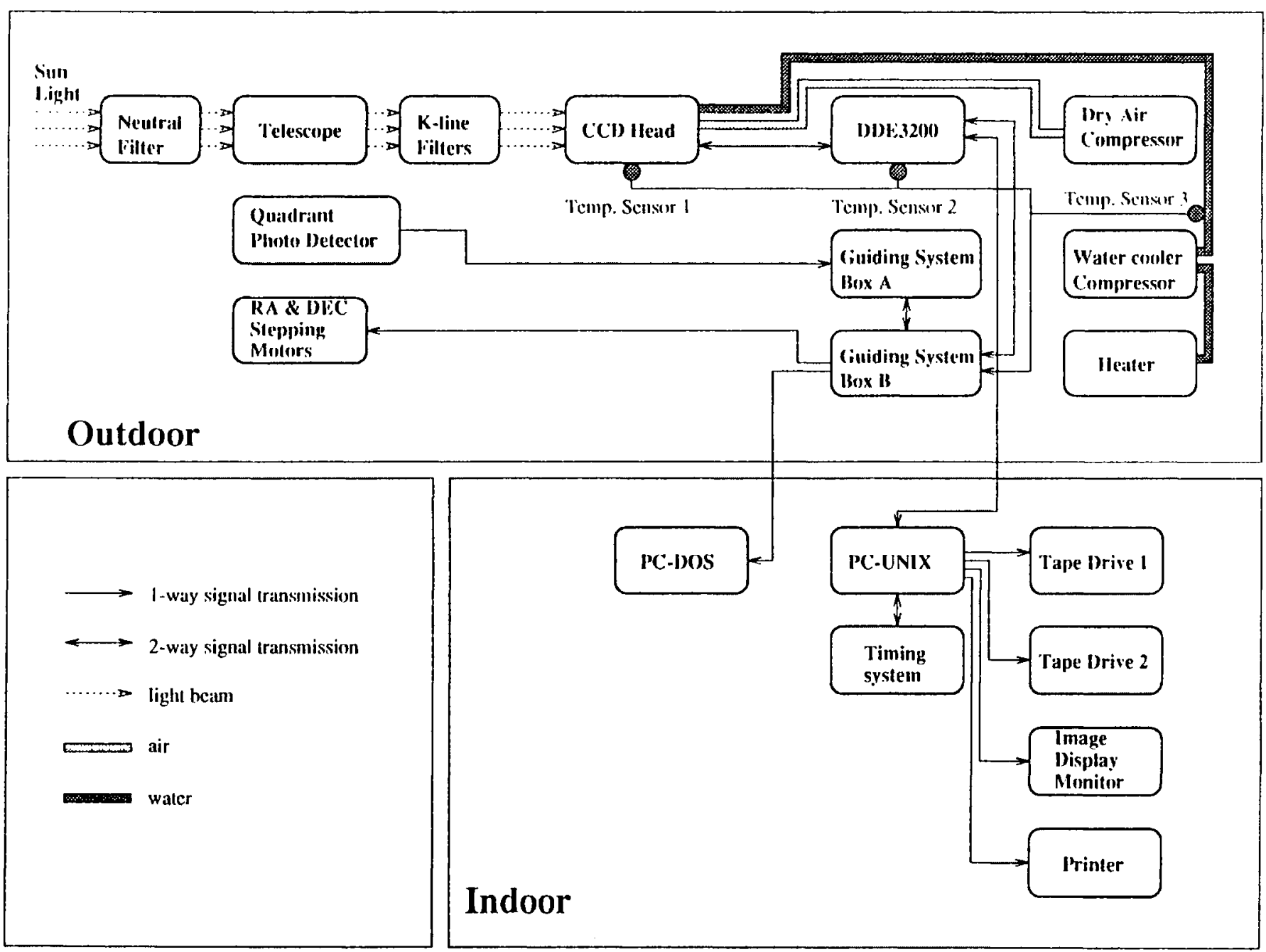

Fig. 1. Block diagram of the TON telescope system. 


\subsection{OPTICAL SYSTEM}

A 3.5-inch ruggedized Questar telescope (Maksutov type) is used. The focus of the telescope can be locked with six screws on three rods attached to the main mirror. The size of the solar disk can vary by changing the separation between the telescope and the CCD. For the present observations, the diameter of the Sun is set to 984 pixels at aphelion so that the annual average is 1000 pixels, corresponding to $22.5 \mathrm{~mm}$ on the CCD detector. It is possible to observe a smaller area on the Sun since solar images can be enlarged by moving the $\mathrm{CCD}$ away from the telescope. A neutral density filter of transmittance $1 / 64$ is used in the front of the telescope to reduce the sunlight. A K-line filter, centered at $3934 \AA$, of FWHM $=10 \AA$ and a prefilter of FWHM $=100 \AA$ are placed right before the CCD detector. A Germantype equatorial mount is used. Two stepping motors of the equatorial mount are controlled by a guiding system to be discussed later in this section.

\subsection{IMAGING SYSTEM}

A 16-bit 1242 by 1152 CCD is used to take images, but only 1080 by 1080 pixels are read out to reduce read-out time such that one image would be taken and saved on magnetic tapes every minute. The CCD is operated at a level of about 240000 electrons per pixel cell to assure a linear response and low photon noise. The analog-digital conversion is set such that one unit corresponds to 6 electrons. The exposure time is set to $800 \mathrm{~ms}$. The CCD is controlled by a PC-486 running UNIX, whose multi-tasking capability is necessary for data acquisition and observing control. The image data are recorded by two 8-mm Exabyte tape drives.

To reduce the thermal noise of the CCD chip, the temperature of the CCD chip is kept at about $223-233 \mathrm{~K}$. Circulating cool water is required to take away the heat from the three-stage thermal electric cooler in the CCD module. Cooling of the body of the CCD module often creates condensation of the moisture in the air on the CCD window. Thus dry air is constantly blown on the window to prevent moisture condensation. For the TON operation, the photon noise, which is about $0.2 \%$, is greater than the thermal noise of the CCD and its circuit. Since the CCD and the circulating water are located outdoors, a heating device is required for sites whose temperature might go below the freezing point to prevent the circulating water from freezing.

\subsection{TELESCOPE GUIDING SYSTEM}

The telescope guiding system is a feed-back system consisting of a guider and an analog computing circuit. The guider consists of a lens which forms a 1-mm solar image on a quadrant photodiode. The analog computing circuit continuously determines the position of the solar image on the photodiode, and its signal is used to drive two stepping motors of the equatorial mount such that the solar image is maintained at the center of the photodiode. The fine adjustment of the alignment 
between the guider and the telescope can be made by changing the offset voltages of the analog computing circuit.

The alignment between the guider and the telescope may slowly change as the telescope moves, which would result in a shift of CCD images up to 30 arc sec over a day. This alignment change is compensated by the following way. The center of every CCD image is computed by software running on the PC (UNIX). The deviation of the center of the CCD images is fed into the analog computing circuit to modify its offset voltages.

\section{Scientific Goals}

A special characteristic of helioseismology is that very rich information on the solar interior can be derived from one set of good data (Deubner and Gough, 1984; Christensen-Dalsgaard et al., 1985; Toomre, 1986; Libbrecht, 1988; Hill, Deubner, and Isaak, 1991; Gough and Toomre, 1991). The TON full-disk images have a spatial sampling window of 1.8 arc sec per pixel. Thus it can provide information of modes up to $l \approx 1000$ with high frequency resolution. Because of the information on the wide range of $l$, the TON data can be used to study many helioseismology problems, including traditional helioseismology problems, such as the inversion of the sound speed, differential rotation, the global magnetic field in the convection zone, as well as problems requiring high- $l$ mode information, such as sunspot helioseismology (Braun, Duvall, and LaBonte, 1987, 1988; Braun, LaBonte, and Duvall, 1990; Braun et al., 1992a, b; Braun, 1995; Davila, 1990; Bogdan, 1992; 1994; Chou et al., 1995), local seismology (Braun et al., 1992a, b; Lindsey, Braun, and Jefferies, 1992), and ring-diagram analysis (Hill, 1988, 1989; Morrow, 1988). The continuous full-disk K-line images can also be used to study some old problems in solar physics, such as the evolution of global magnetic fields and the rotation speed at high altitudes.

\section{Preliminary Results}

In this paper, we show an $l-\nu$ diagram derived from 512 images, taken at Teide Observatory on February 16, 1994, to give an idea about the quality of the TON data. The first step in the data reduction is the removal of the dark current, flatfielding, and registration. Dark-current images were taken with the telescope cover on. Flat images were taken by replacing the neutral filter in front of the telescope by a ground glass. The radial variation of flat image is corrected. The average intensity over the solar disc is normalized to remove the variation of atmospheric transparency, though the information of low- $l$ modes is lost. A two-point filter is applied to remove the limb darkening, solar activity, convection, and atmospheric variation. The spatial mean of each image is subtracted to remove the leakage from 


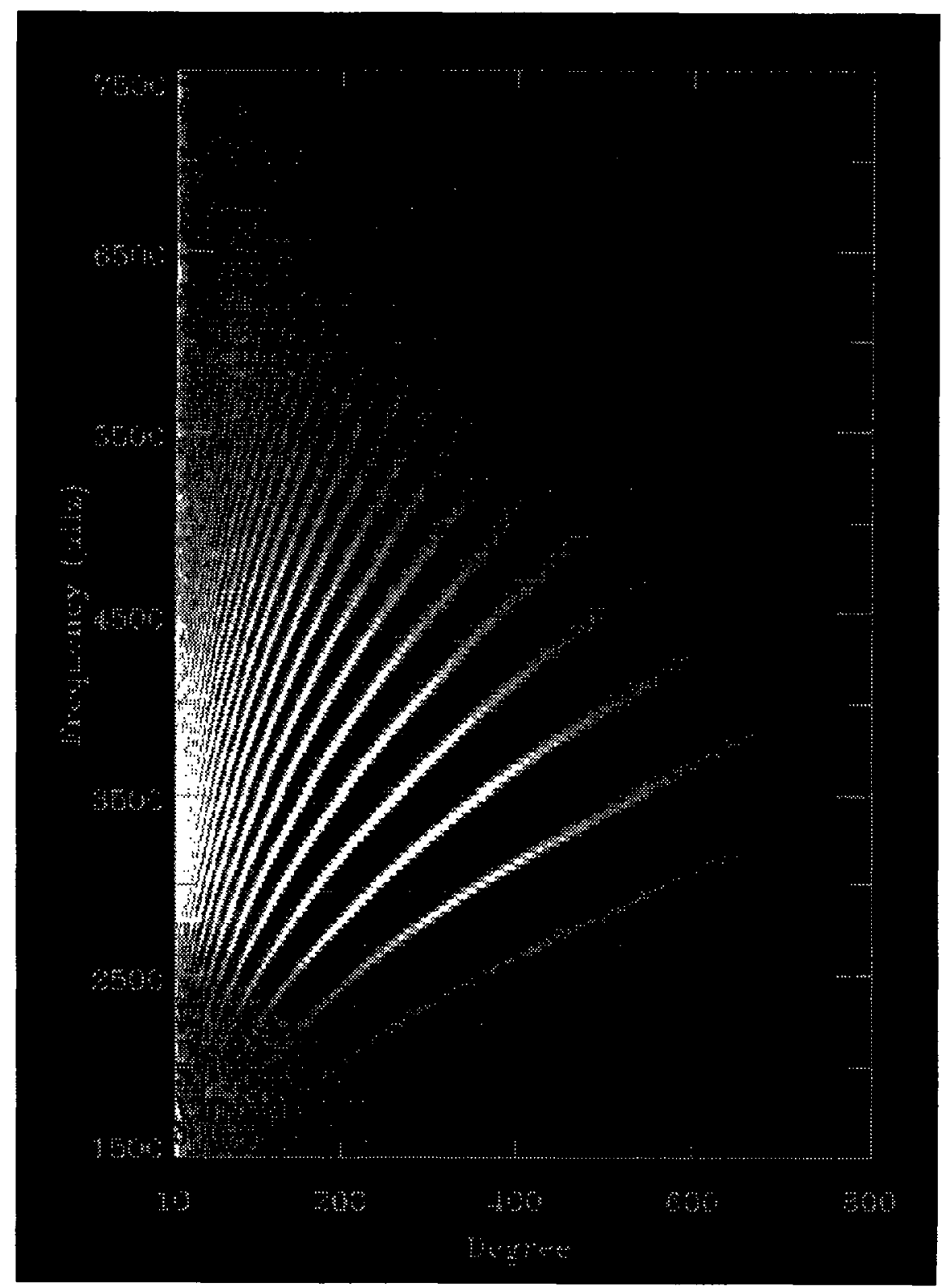

Fig. 2. $l-\nu$ diagram constructed from 512 images.

the $l=0$ mode to the adjacent modes. The software package GRASP, developed by GONG, is used to compute the coefficients of spherical harmonic expansion and do the temporal Fourier transform. The rotation is corrected and then the $l-\nu$ diagram is constructed by averaging the power over the azimuthal order $m$. The $l-\nu$ diagram is shown in Figure 2. It is noted that the effect of the two-point filter is removed in the $\nu$ domain. Figure 2 shows that the $p$-mode ridges can easily be identified up to $l=800$. 


\section{Acknowledgements}

The TON project is supported by NSC of ROC under grants NSC-83-NSPO-BRDD-007-01 and NSC-84-2612-M-007-001. MTS is also supported by NSC-84 -2612-M-182-001. AJ and MCR are supported by Spanish CICYT under grants ESP 90-0969 and PB 91-0530; MCR receives partial support from the Brazilian Institution: CNPq (202504/91-3). We deeply thank all observers who were dedicated to this long-term observation. We are grateful to the GONG Data Team, especially to Drs E. R. Anderson, F. Hill, and J. Leibacher, for providing the software and helping in the data reduction.

\section{References}

Bogdan, T. J.: 1992, in J. H. Thomas and N. O. Weiss (eds.), Sunspots: Theory and Observations, Kluwer Academic Publishers, Dordrecht, Holland, p. 345.

Bogdan, T. J.: 1994, in N. Schmitt and M. Schussler (eds.), Solar Magnetic Fields, Cambridge University Press, Cambridge.

Braun, D. C.: 1995, Astrophys. J., in press.

Braun, D. C., Duvall, T. L., Jr., and LaBonte, B. J.: 1987, Astrophys. J. 319, L27.

Braun, D. C., Duvall, T. L., Jr., and LaBonte, B. J.: 1988, Astrophys. J. 335, 1015.

Braun, D. C., LaBonte, B. J., and Duvall, T. L., Jr.: 1990, Astrophys. J. 354, 372.

Braun, D. C., Duvall, T. L., Jr., LaBonte, B. J., Jefferies, S. M., Harvey, J. W., and Pomerantz, M. A.: 1992a, Astrophys. J. 391, L113.

Braun, D. C., Lindsey, C., Fan, Y., and Jefferies, S. M.: 1992b, Astrophys. J. 392, 739.

Chou, D.-Y., Chou, H.-Y., Hsieh, Y.-C., and Chen, C.-K.: 1995, Astrophys. J., submitted.

Christensen-Dalsgaard, J., Duvall, T. L., Jr., Gough, D. O., Harvey, J. W., and Rhodes, E. J., Jr.: 1985, Nature 315, 378.

Davila, J. M.: 1990, in E. R. Priest and V. Krishan (eds.), Basic Plasma Processes on the Sun, D. Reidel Publ. Co., Dordrecht, Holland, p. 149.

Deubner, F. L. and Gough, D.: 1984, Ann. Rev. Astron. Astrophys. 22, 593.

Duvall, T. L., Jr., Jefferies, S. M., Harvey, J. W., Osaki, Y., and Pomerantz, M. A.: 1993, Astrophys. J. 410, 829 .

Elsworth, Y., Elowe, R., Isaak, G. R., McLeod, C. P., Miller, B. A., Wheeler, S. J., and New, R.: 1994, GONG 1994: Helio- and Astero-Seismology from Earth and Space, in press.

Fossat, E.: 1991, Solar Phys. 133, 1.

Gough, D. and Toomre, J.: 1991, Ann. Rev. Astron. Astrophys. 29, 627.

Harvey, J. and the GONG Instrument Development Team: 1988, in E. J. Rolfe (ed.), Seismology of the Sun and Sun-Like Stars, ESA SP-286, p. 203.

Hill, F.: 1988, Astrophys. J. 333, 996.

Hill, F.: 1989, Astrophys. J. 343, L69.

Hill, F., Deubner, F.-L., and Isaak, G. R.: 1991, in A. N. Cox, W. C. Livingston, and M. S. Matthews (eds.), Solar Interior and Atmosphere, University of Arizona Press, Tucson, p. 329.

Libbrecht, K. G.: 1988, Space Sci. Rev. 47, 275.

Lindsey, C., Braun, D. C., and Jefferies, S. M.: 1992, in T. M. Brown (ed.), GONG 1992: Seismic Investigation of the Sun and Stars, Astron. Soc. Pacific Conf. Proc., p. 81.

Morrow, C. A.: 1988, Ph.D. Thesis, University of Colorado.

Ronan, R. S. and LaBonte, B. J.: 1994, Solar Phys. 149, 1.

Toomre, J.: 1986, in D. O. Gough (ed.), Seismology of the Sun and the Distant Stars, D. Reidel Publ. Co., Dordrecht, Holland, p. 1. 